\title{
An approach for the state estimation of Takagi-Sugeno models and application to sensor fault diagnosis
}

\author{
Dalil Ichalal, Benoît Marx, José Ragot, Didier Maquin
}

\begin{abstract}
In this paper, a new method to design an observer for nonlinear systems described by Takagi-Sugeno (TS) model, with unmeasurable premise variables, is proposed. Most of existing work on TS models consider models with measurable decision variables. As a consequence, these works cannot be applied when the decision variables are not available to measurement. The idea of the proposed approach is to rewrite the TS model with unmeasurable premise variable into an uncertain TS model by introducing the estimated state in the model. The convergence of the state estimation error is studied using the Lyapunov theory and the stability conditions are given in terms of Linear Matrix Inequalities (LMIs). Finally, an academic example is given to illustrate the proposed approach, with an application to sensor fault detection and isolation using an observer bank.

Index Terms-Nonlinear systems, Takagi-Sugeno models, state estimation, unmeasurable premise variable, uncertain systems, $\mathcal{L}_{2}$ optimization, sensor fault diagnosis.
\end{abstract}

\section{INTRODUCTION}

The Takagi-Sugeno (TS) fuzzy model is a popular and important modeling framework due, on the one hand, to its ability to represent with a good precision a large class of nonlinear systems and, on the other hand, to its ease of manipulation from the mathematical point of view compared to the original nonlinear models. In the literature, increasing attention has been devoted to TS fuzzy models specially in the fields of control, state estimation and diagnosis of nonlinear systems.

The problem of state estimation of nonlinear systems can be viewed as the heart of control systems and modelbased diagnosis. In [7] and [16], quadratic and non-quadratic stability conditions for TS fuzzy models are established using the Lyapunov theory and the Linear Matrix Inequality formalism (LMI). In [12] and [15], the authors proposed less conservative conditions for ensuring the stability and a method for designing a feedback control law to stabilize TS systems. The problem of model-based diagnosis has been studied in [1], [10] and [13].

In the literature, only a few works dealt with the problem of state estimation of nonlinear systems described by TS models with unmeasurable premise variables. In [3] and [4], the Thau-Luenberger observer was extended to this class of systems. The authors of [5] proposed a sliding mode observer for TS systems with unmeasurable premise variables. The problem which can be pointed out is the fact that the

All the authors are with the Centre de Recherche en Automatique de Nancy (CRAN), Nancy-Université, 2, avenue de la forêt de Haye 54516 Vandoeuvre-les-Nancy \{dalil.ichalal, benoit.marx, jose.ragot, didier.maquin\}eensem. inpl-nancy. fr convergence conditions used Lipschitz conditions which are very restrictive.

In this paper, another method is proposed by using a TS model approach to design observers for nonlinear systems. We consider the case where the weighting functions depend on unmeasurable decision variables (the state of the system). Section II introduces the problem and some background. In section III, the main results to design the observer are given under LMI formulation. The idea is to transform the TS system with unmeasurable premise variable into an uncertain TS system with estimated premise variable. And by using the Lyapunov theory and the $\mathcal{L}_{2}$ optimization techniques, the convergence conditions are proposed. Finally, in section IV, a simulation example is given for state estimation and sensor fault detection and isolation.

\section{NOTATIONS AND PROBLEM STATEMENT}

\section{A. Takagi-Sugeno structure}

Consider a nonlinear system described by

$$
\left\{\begin{array}{l}
\dot{x}(t)=f(x, u) \\
y(t)=h(x, u)
\end{array}\right.
$$

The TS fuzzy modeling allows to represent the behavior of a nonlinear system (1) by the interpolation of a set of linear sub-models. Each sub-model contributes to the global behavior of the nonlinear system through a weighting function $\mu_{i}(\xi(t))$. The TS structure is given by

$$
\left\{\begin{array}{l}
\dot{x}(t)=\sum_{i=1}^{r} \mu_{i}(\xi(t))\left(A_{i} x(t)+B_{i} u(t)\right) \\
y(t)=\sum_{i=1}^{r} \mu_{i}(\xi(t))\left(C_{i} x(t)+D_{i} u(t)\right)
\end{array}\right.
$$

where $x(t) \in \mathbb{R}^{n}$ is the state vector, $u(t) \in \mathbb{R}^{m}$ is the input vector, $y(t) \in \mathbb{R}^{p}$ represents the output vector. $A_{i} \in \mathbb{R}^{n \times n}$, $B_{i} \in \mathbb{R}^{n \times m}, C_{i} \in \mathbb{R}^{p \times n}$ and $D_{i} \in \mathbb{R}^{p \times m}$ are known matrices. Finally, the functions $\mu_{i}(\xi(t))$ are the weighting functions depending on the variables $\xi(t)$ which can be measurable (as the input or the output of the system) or non measurable variables (as the state of the system). These functions verify the following properties

$$
\left\{\begin{array}{l}
\sum_{i=1}^{r} \mu_{i}(\xi(t))=1 \\
0 \leq \mu_{i}(\xi(t)) \leq 1 \quad \forall i \in\{1,2, \ldots, r\}
\end{array}\right.
$$

In order to obtain a TS model (2) from (1) different methods exist such as linearizing the equation (1) around some operating points and using adequate weighting functions. The most interesting and important way to obtain a TS model 
is the well-known transformation by nonlinear sector [17]. Indeed, this transformation allows to obtain an exact TS representation of (1). It is proved in [18] that if an output affected by disturbance (which cannot be avoided in practical situations) is considered as a decision variable, the obtained TS system does not represent precisely the model given by (1) of a nonlinear system. It is also pointed out that if the output is nonlinear with respect to the state of the system it is difficult or even impossible to obtain a TS model by nonlinear sector transformation with the output as a premise variable. These facts motivate the will to take the state of the system as premise variable in order to describe a wider class of nonlinear systems.

The use of TS fuzzy models allows to generalize some tools developed in the linear domain to the nonlinear systems. This representation is very interesting in the sense that it simplifies the stability studies of nonlinear systems and the design of control laws and observers. In [7], [11], [12], the stability and stabilization tools are inspired from the study of linear systems. In [2], [13], the authors worked on the problem of state estimation and application for diagnosis of TS fuzzy systems. The proposed approaches in these last papers are the generalization of the classical observers (Luenberger Observer and Unknown Input Observer (UIO)) to the nonlinear domain.

\section{B. Problem statement}

In the context of fault diagnosis of nonlinear systems by TS approach, the problem of fault isolation is not possible with only one model. Indeed, if the actuator fault isolation problem is considered, constructing a bank of observers in order to isolate faults is not possible because if the $i^{\text {th }}$ input is used as a premise variable, then all the observers considering that the $i^{\text {th }}$ input, then the state estimation is not decoupled from this input. The same problem is encountered when trying to isolate the sensor faults with a TS model which output is the premise variable. The solution for this problem which is largely used in the literature is to develop two different TS models for the same nonlinear system. The first TS model uses the input of the system as premise variable in order to isolate sensor faults. In the second one, dedicated to actuator fault isolation, the output of the system is the premise variable. To overcome this difficulty, the proposed solution is to develop only one TS model which uses the state of the system as premise variable for the nonlinear system; then the problems of actuator and sensor fault isolation can be simultaneously solved. In fact, one of the key points in the diagnosis procedure is the observer design.

The TS model structure also appears in the framework of cryptanalysis and chaotic systems. In [8], based on these models and considering that the output of the system is the premise variable, a new observer design method is proposed in order to achieve synchronization. It is pointed out that using the unknown state as a premise variable will improve the synchronization process security.

\section{Notations and preliminaries}

The considered systems are those described by the equation (2) with weighting functions depending on the state of the system. In most of the practical situation, the sensor location does not depend on the operating point. As a consequence, considering $C_{1}=C_{2}=\ldots=C$ is realistic. In order to ease the calculus, no direct transfer from $u(t)$ to $y(t)$ is considered but the case $D \neq 0$ can be dealt with similarly. The system (2) becomes

$$
\left\{\begin{array}{l}
\dot{x}(t)=\sum_{i=1}^{r} \mu_{i}(x(t))\left(A_{i} x(t)+B_{i} u(t)\right) \\
y(t)=C x(t)
\end{array}\right.
$$

which can be re-written in the form

$$
\begin{aligned}
\dot{x}(t) & =\sum_{i=1}^{r}\left(\mu_{i}(\hat{x}(t))\left(A_{i} x(t)+B_{i} u(t)\right)\right. \\
& \left.+\delta_{i}(t)\left(A_{i} x(t)+B_{i} u(t)\right)\right)
\end{aligned}
$$

where

$$
\delta_{i}(t)=\mu_{i}(x(t))-\mu_{i}(\hat{x}(t))
$$

Let us define

$$
\begin{aligned}
\Delta A(t) & =\sum_{i=1}^{r} \delta_{i}(t) A_{i} \\
& =\mathcal{A} \Sigma_{A}(t) E_{A} \\
\Delta B(t) & =\sum_{i=1}^{r} \delta_{i}(t) B_{i} \\
& =\mathcal{B} \Sigma_{B}(t) E_{B}
\end{aligned}
$$

where

$$
\begin{gathered}
\mathcal{A}=\left[\begin{array}{lll}
A_{1} & \cdots & A_{r}
\end{array}\right], E_{A}=\left[\begin{array}{lll}
I_{n} & \ldots & I_{n}
\end{array}\right]^{T} \\
\Sigma_{A}(t)=\left[\begin{array}{ccc}
\delta_{1}(t) I_{n} & \cdots & 0 \\
\vdots & \ddots & \vdots \\
0 & \cdots & \delta_{r}(t) I_{n}
\end{array}\right] \\
\mathcal{B}=\left[\begin{array}{llll}
B_{1} & \cdots & B_{r}
\end{array}\right], E_{B}=\left[\begin{array}{lll}
I_{m} & \ldots & I_{m}
\end{array}\right]^{T} \\
\Sigma_{B}(t)=\left[\begin{array}{ccc}
\delta_{1}(t) I_{m} & \cdots & 0 \\
\vdots & \ddots & \vdots \\
0 & \cdots & \delta_{r}(t) I_{m}
\end{array}\right]
\end{gathered}
$$

The convex sum property of the weighting functions allows to write

$$
-1 \leq \delta_{i}(t) \leq 1
$$

then

$$
\begin{aligned}
& \Sigma_{A}^{T}(t) \Sigma_{A}(t) \leq I \\
& \Sigma_{B}^{T}(t) \Sigma_{B}(t) \leq I
\end{aligned}
$$

The system (5) becomes

$$
\left\{\begin{aligned}
\dot{x}(t) & =\sum_{i=1}^{r} \mu_{i}(\hat{x}(t))\left(\left(A_{i}+\Delta A(t)\right) x(t)\right. \\
& \left.+\left(B_{i}+\Delta B(t)\right) u(t)\right) \\
y(t) & =C x(t)
\end{aligned}\right.
$$

Finally, the system (4) with unmeasurable premise variable is transformed into an equivalent uncertain TS model with known premise variable (9). 


\section{OBSERVER DESIGN}

For the uncertain model (9), the following observer is proposed

$$
\begin{aligned}
\dot{\hat{x}}(t) & =\sum_{i=1}^{r} \mu_{i}(\hat{x}(t))\left(A_{i} \hat{x}(t)+B_{i} u(t)+L_{i}(y(t)-\hat{y}(t))\right) \\
\hat{y}(t) & =C \hat{x}(t)
\end{aligned}
$$

The gains $L_{i}$ must be determined to ensure the asymptotic convergence of the estimated state $\hat{x}$ to the actual state of the system $x$. In the sequel, for the sake of simplicity, the time variable $t$ will be omitted.

Let us define the state estimation error

$$
e=x-\hat{x}
$$

ruled by the following equation

$$
\dot{e}=\sum_{i=1}^{r} \mu_{i}(\hat{x})\left(\left(A_{i}-L_{i} C\right) e\right)+\Delta A x+\Delta B u
$$

Note that the dynamic equation describing the state estimation error depends on the input $u(t)$ and on the state $x(t)$. Then the problem of designing the observer (10) reduces to finding the gains $L_{i}$ in order that the system (12) generating $e(t)$ is stable and that the influence of $u(t)$ on $e(t)$ is minimized.

Let us define the augmented vector $e_{a}=\left[\begin{array}{ll}e^{T} & x^{T}\end{array}\right]^{T}$, from which the following augmented system is obtained

$$
\dot{e}_{a}=\sum_{i=1}^{r} \sum_{j=1}^{r} \mu_{i}(\hat{x}) \mu_{j}(x)\left(\bar{A}_{i j} e_{a}+\bar{B}_{i j} u\right)
$$

where

$$
\bar{A}_{i j}=\left[\begin{array}{cc}
\Phi_{i} & \Delta A \\
0 & A_{j}
\end{array}\right], \bar{B}_{i j}=\left[\begin{array}{c}
\Delta B \\
B_{j}
\end{array}\right], \Phi_{i}=A_{i}-L_{i} C
$$

Theorem 1: The system (12) is stable and the $\mathcal{L}_{2}$ gain of the transfer from $u(t)$ to the state estimation error is bounded by $\gamma$, if there exists two positive and symmetric matrices $P_{1}$ and $P_{2}$, matrices $K_{i}$, and positive scalars $\lambda_{1}, \lambda_{2}$ and $\bar{\gamma}$ such that the following LMIs hold $\forall i, j \in\{1, \ldots, r\}$

$$
\left[\begin{array}{ccccc}
\Psi_{i} & 0 & 0 & P_{1} \mathcal{A} & P_{1} \mathcal{B} \\
0 & \Xi_{j} & P_{2} B_{j} & 0 & 0 \\
0 & B_{j}^{T} P_{2} & -\bar{\gamma} I+\lambda_{2} E_{B}^{T} E_{B} & 0 & 0 \\
\mathcal{A}^{T} P_{1} & 0 & 0 & -\lambda_{1} I & 0 \\
\mathcal{B}^{T} P_{1} & 0 & 0 & 0 & -\lambda_{2} I
\end{array}\right]_{(15)}
$$

where

$$
\begin{gathered}
\Psi_{i}=A_{i}^{T} P_{1}+P_{1} A_{i}-K_{i} C-C^{T} K_{i}^{T}+I \\
\Xi_{j}=A_{j}^{T} P_{2}+P_{2} A_{j}+\lambda_{1} E_{A}^{T} E_{A}
\end{gathered}
$$

The gains of the observer are computed from

$$
L_{i}=P_{1}^{-1} K_{i}
$$

$\mathcal{L}_{2}$-gain from $u(t)$ to $e(t)$ is obtained by

$$
\gamma=\sqrt{\bar{\gamma}}
$$

Proof: The proof of the theorem 1 is established by using the following quadratic Lyapunov function candidate

$$
V=e_{a}^{T} P e_{a}, \quad P=P^{T}>0
$$

Its derivative with regard to time is given by

$$
\dot{V}=\dot{e}_{a}^{T} P e_{a}+e_{a}^{T} P \dot{e}_{a}
$$

By using the dynamic of the state estimation error (13), the following is obtained

$$
\begin{aligned}
\dot{V} & =\sum_{i=1}^{r} \sum_{j=1}^{r} \mu_{i}(\hat{x}) \mu_{j}(x)\left(e_{a}^{T} \bar{A}_{i j}^{T} P e_{a}+e_{a}^{T} P \bar{A}_{i j} e_{a}\right. \\
& \left.+u^{T} \bar{B}_{i j}^{T} P e_{a}+e_{a}^{T} P \bar{B}_{i j} u\right)
\end{aligned}
$$

The state estimation error is the output of (13) defined by

$$
e(t)=H e_{a}
$$

where

$$
H=\left[\begin{array}{ll}
I & 0
\end{array}\right]
$$

The system (12) is stable and the gain $\mathcal{L}_{2}$ of the transfer from $u(t)$ to $e(t)$ is bounded by $\gamma$ if the following condition holds [6]

$$
\dot{V}+z^{T} z-\gamma^{2} u^{T} u<0
$$

By substituting (22) and (23) in (25), the following inequality is obtained

$$
\begin{aligned}
& \sum_{i=1}^{r} \sum_{j=1}^{r} \mu_{i}(\hat{x}) \mu_{j}(x)\left(e_{a}^{T} \bar{A}_{i j}^{T} P e_{a}+e_{a}^{T} P \bar{A}_{i j} e_{a}\right. \\
&\left.+\quad u^{T} \bar{B}_{i j}^{T} P e_{a}+e_{a}^{T} P \bar{B}_{i j} u+e_{a}^{T} H^{T} H e_{a}-\gamma^{2} u^{T} u\right)<0
\end{aligned}
$$

or equivalently

$\sum_{i=1}^{r} \sum_{j=1}^{r} \mu_{i}(\hat{x}) \mu_{j}(x)\left[\begin{array}{c}e_{a} \\ u\end{array}\right]^{T}\left[\begin{array}{cc}X_{i j} & P \bar{B}_{i j} \\ \bar{B}_{i j}^{T} P & -\gamma^{2} I\end{array}\right]\left[\begin{array}{c}e_{a} \\ u\end{array}\right]<0$

where

$$
X_{i j}=\bar{A}_{i j}^{T} P+P \bar{A}_{i j}+H^{T} H
$$

According to the convex sum property of the weighting functions $\mu_{i}$, the inequality (27) holds if (29) is satisfied

$$
\left[\begin{array}{cc}
\bar{A}_{i j}^{T} P+P \bar{A}_{i j}+H^{T} H & P \bar{B}_{i j} \\
\bar{B}_{i j}^{T} P & -\gamma^{2} I
\end{array}\right]<0, \quad \forall i, j \in\{1, . ., r\}
$$

Let us consider the following particular form of the matrix $P$

$$
P=\left[\begin{array}{cc}
P_{1} & 0 \\
0 & P_{2}
\end{array}\right]
$$

By substituting (14), (24) and (30), equation (29) can be written as

$$
\left[\begin{array}{ccc}
\Phi_{i}^{T} P_{1}+P_{1} \Phi_{i}+I & P_{1} \Delta A & P_{1} \Delta B \\
\Delta A^{T} P_{1} & A_{j}^{T} P_{2}+P_{2} A_{j} & P_{2} B_{j} \\
\Delta B^{T} P_{1} & B_{j}^{T} P_{2} & -\gamma^{2} I
\end{array}\right]<0
$$

Notice that the inequality (31) is time-dependent due to the terms $\Delta A(t)$ and $\Delta B(t)$. However, these latter are bounded. 
Firstly let us re-write (31) separating the time-dependent terms.

$$
\begin{aligned}
{\left[\begin{array}{ccc}
\Phi_{i}^{T} P_{1}+P_{1} \Phi_{i}+I & 0 & 0 \\
0 & A_{j}^{T} P_{2}+P_{2} A_{j} & P_{2} B_{j} \\
0 & B_{j}^{T} P_{2} & -\gamma^{2} I
\end{array}\right] } \\
+\underbrace{\left[\begin{array}{ccc}
0 & P_{1} \Delta A & P_{1} \Delta B \\
\Delta A^{T} P_{1} & 0 & 0 \\
\Delta B^{T} P_{1} & 0 & 0
\end{array}\right]}_{\mathcal{W}}<0
\end{aligned}
$$

The time-dependent matrix $\mathcal{W}$ is decomposed as follows

$$
\mathcal{W}=\mathcal{Q}+\mathcal{Q}^{T}
$$

where

$$
\mathcal{Q}=\left[\begin{array}{ccc}
0 & P_{1} \Delta A & P_{1} \Delta B \\
0 & 0 & 0 \\
0 & 0 & 0
\end{array}\right]
$$

According to the definition of $\Delta A(t)$ and $\Delta B(t)$ given in (6) and (7), the matrix $\mathcal{Q}$ is written as follows

$$
\mathcal{Q}=\left[\begin{array}{cc}
P_{1} \mathcal{A} & P_{1} \mathcal{B} \\
0 & 0 \\
0 & 0
\end{array}\right]\left[\begin{array}{ccc}
0 & \Sigma_{A}(t) E_{A} & 0 \\
0 & 0 & \Sigma_{B}(t) E_{B}
\end{array}\right]
$$

To be able to bound $\mathcal{W}$, let us introduce the following lemma

Lemma 1: Consider $X$ and $Y$ with appropriate dimensions and $\Omega$ a positive definite matrix. the following property is verified

$$
X^{T} Y+Y^{T} X \leq X^{T} \Omega X+Y^{T} \Omega^{-1} Y \quad \Omega>0
$$

Applying this Lemma to $\mathcal{W}$ (33) with $\Omega$ defined as follows

$$
\Omega=\left[\begin{array}{cc}
\lambda_{1} I & 0 \\
0 & \lambda_{2} I
\end{array}\right]
$$

we obtain

$$
\mathcal{W} \leq \Theta \Omega^{-1} \Theta^{T}+\Psi^{T} \Omega \Psi
$$

where

$$
\begin{gathered}
\Theta=\left[\begin{array}{cc}
P_{1} \mathcal{A} & P_{1} \mathcal{B} \\
0 & 0 \\
0 & 0
\end{array}\right] \\
\Psi=\left[\begin{array}{ccc}
0 & \Sigma_{A}(t) E_{A} & 0 \\
0 & 0 & \Sigma_{B}(t) E_{B}
\end{array}\right]
\end{gathered}
$$

After some computations using the properties of $\Sigma_{A}(t)$ (8) and $\Sigma_{B}(t)(8)$, we obtain

$$
\mathcal{W} \leq\left[\begin{array}{ccc}
Y & 0 & 0 \\
0 & \lambda_{1} E_{A}^{T} E_{A} & 0 \\
0 & 0 & \lambda_{2} E_{B}^{T} E_{B}
\end{array}\right]
$$

where

$$
Y=\lambda_{1}^{-1} P_{1} \mathcal{A} \mathcal{A}^{T} P_{1}+\lambda_{2}^{-1} P_{1} \mathcal{B B}^{T} P
$$

Substituting $\mathcal{W}(40)$ in (32), the following is obtained

$$
\left[\begin{array}{ccc}
\Xi & 0 & 0 \\
0 & \Xi_{j} & P_{2} B_{j} \\
0 & B_{j}^{T} P_{2} & -\gamma^{2} I+\lambda_{2} E_{B}^{T} E_{B}
\end{array}\right]<0
$$

with

$$
\Xi=\Phi_{i}^{T} P_{1}+P_{1} \Phi_{i}+\lambda_{1}^{-1} P_{1} \mathcal{A} \mathcal{A}^{T} P_{1}+\lambda_{2}^{-1} P_{1} \mathcal{B B}^{T} P_{1}+I
$$

The matrix inequality (42) is not linear with regard to the variables $P_{1}, P_{2}, L_{i}, \lambda_{1}, \lambda_{3}$ and $\gamma$. In order to solve these matrix inequalities, it is necessary to linearize them to obtain LMIs. To do this, we will use the Schur complement and some variable changes: $K_{i}=P_{1} L_{i}$ and $\bar{\gamma}=\gamma^{2}$ which allows to obtain (15).

Then, the convergence conditions of the state estimation error are given in Linear Matrix Inequalities (LMIs) in the theorem 1 .

Remark 1: Form the theorm 1, the $\mathcal{L}_{2}$ gain of the transfer from $u(t)$ to $e(t)$ is chosen before the computation of LMIs (15). In order to increase the quality of the state reconstruction and obtaining an optimal observer, it is possible to minimize the $\mathcal{L}_{2}$ gain $\gamma$ of the transfer from $u(t)$ to $e(t)$. Then, the problem to solve becomes

$$
\min _{P_{1}, P_{2}, K_{i}, \lambda_{1}, \lambda_{2}} \bar{\gamma} \quad \text { s.t. (15) }
$$

\section{APPLICATION TO SENSOR FAULT ISOLATION}

The proposed observer can be used in order to construct a scheme for sensor fault detection and isolation (FDI) of a nonlinear system represented by a TS fuzzy model. The measured output is defined by $\tilde{y}(t)$ :

$$
\tilde{y}(t)=y(t)+f(t)+w(t)
$$

where $f(t)$ represents the sensor fault vector and $w(t)$ a zeromean noise vector. Several fault detection methods based on the state estimation allow the sensor fault detection and isolation (see, for example, [9] and [14] for further details about these methods). Classic observer schemes (for example, Dedicated Observer Scheme (DOS) or Generalized Observer Scheme (GOS)) can be employed in order to generate faults accentuated signals also called residual signals.

In this paper, the scheme represented in the figure 1. is used, for a system with two outputs. Each of the two observers used one of the two outputs; that allows to generate four residuals.

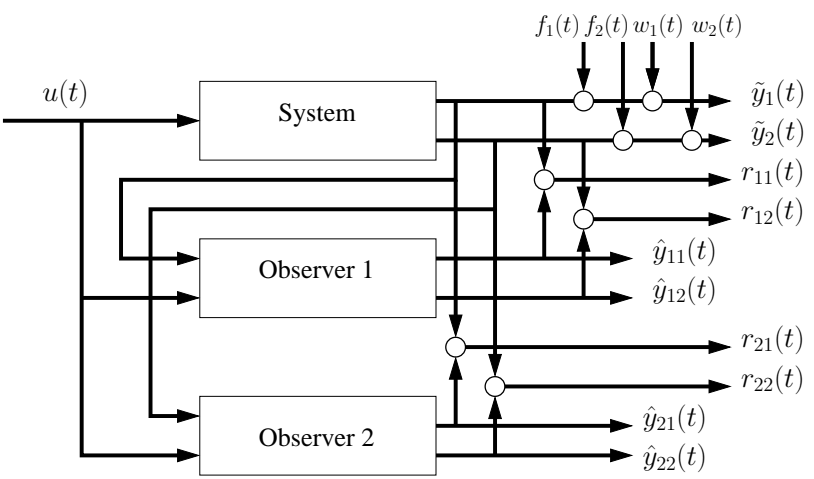

Fig. 1. Scheme for sensor fault detection and isolation

A theoretic decision logic is developed to see how the faults affect the residual signals which are defined as follows

$$
r_{i j}(t)=\tilde{y}_{j}(t)-\hat{y}_{i j}(t)
$$


where $\tilde{y}_{j}(t)$ are the components of $\tilde{y}(t)$ and $\hat{y}_{i j}$ are the components of the reconstructed outputs with the $i^{t h}$ observer.

$$
1 \leq i \leq 2, \quad 1 \leq j \leq 2
$$

$i$ represents the observer number and $j$ the output number. The output $\tilde{y}_{1}(t)$ is affected by the fault $f_{1}(t)$ and the outputs

\begin{tabular}{|l|l|l|l|l|}
\cline { 2 - 5 } \multicolumn{1}{c|}{} & $r_{11}(t)$ & $r_{12}(t)$ & $r_{21}(t)$ & $r_{22}(t)$ \\
\hline$f_{1}(t)$ & $?$ & $?$ & 1 & 0 \\
\hline$f_{2}(t)$ & 0 & 1 & $?$ & $?$ \\
\hline
\end{tabular}

TABLE I

DECISION LOGIC

$\tilde{y}_{2}(t)$ is fault free. Consequently, the observer 1 is corrupted by the fault $f_{1}(t)$ and the residual signals $r_{1 j}(t)$ can be different from zero. However, the fact that the system is nonlinear, a compensation phenomenons can appear and the influence of the fault can be masked on the residuals, then, in the incidence table, "?" is used to say that no decision can be taken. But, the state reconstruction given by the observer 2 is correct because this observer uses the fault free output $y_{2}(t)$. Therefore, the residual signals $r_{2 j}$ are sensitive to the fault $f_{1}(t)$ which represented by " 1 " in the incidence table. A similar reasoning can be used to detect the fault $f_{2}(t)$.

\section{EXAMPLE}

\section{A. State estimation}

Let us consider the system (4) defined by the following matrices

$$
\begin{aligned}
& A_{1}=\left[\begin{array}{ccc}
-2 & 1 & 1 \\
1 & -3 & 0 \\
2 & 1 & -8
\end{array}\right], A_{2}=\left[\begin{array}{ccc}
-3 & 2 & -2 \\
5 & -3 & 0 \\
1 & 2 & -4
\end{array}\right] \\
& B_{1}=\left[\begin{array}{c}
1 \\
0.5 \\
0.5
\end{array}\right], B_{2}=\left[\begin{array}{c}
0.5 \\
1 \\
0.25
\end{array}\right], C=\left[\begin{array}{llc}
1 & 1 & 1 \\
1 & 0 & 1
\end{array}\right]
\end{aligned}
$$

The weighting functions are defined by:

$$
\left\{\begin{array}{l}
\mu_{1}(x)=\frac{1-\tanh \left(x_{1}\right)}{2} \\
\mu_{2}(x)=1-\mu_{1}(x)=\frac{1+\tanh \left(x_{1}\right)}{2}
\end{array}\right.
$$

Minimizing the gain $\bar{\gamma}$ of the transfer from $u(t)$ to the state estimation error $e(t)$ subject to the LMIs given in the theorem 1 , gives the following results

$$
\begin{gathered}
L_{1}=\left[\begin{array}{cc}
-35.66 & 121.56 \\
57.61 & 63.59 \\
15.22 & -9.55
\end{array}\right], L_{2}=\left[\begin{array}{cc}
-37.01 & 121.47 \\
56.10 & 68.80 \\
-0.25 & -6.09
\end{array}\right], \\
P_{1}=\left[\begin{array}{ccc}
0.05 & -0.03 & 0.07 \\
-0.03 & 0.4 & -0.06 \\
0.07 & -0.06 & 0.3
\end{array}\right], \\
P_{2}=\left[\begin{array}{ccc}
3.01 & 1.11 & 0.35 \\
1.11 & 2.34 & 0.07 \\
0.35 & 0.07 & 1.09
\end{array}\right], \\
\lambda_{1}=3.47, \lambda_{2}=0.0028 \times 10^{-5}, \gamma=0.0894
\end{gathered}
$$

The state estimation error converges (see figure 1) and the gain of the transfer from $u(t)$ to $e(t)$ is bounded by $\gamma=$ 0.0894 since the input $u(t)$ (see fig. 2) is bounded by 1 , the state estimation error is bounded by $\gamma=0.0894$ that may be considered as acceptable when considering the magnitude of the state (see fig. 4).

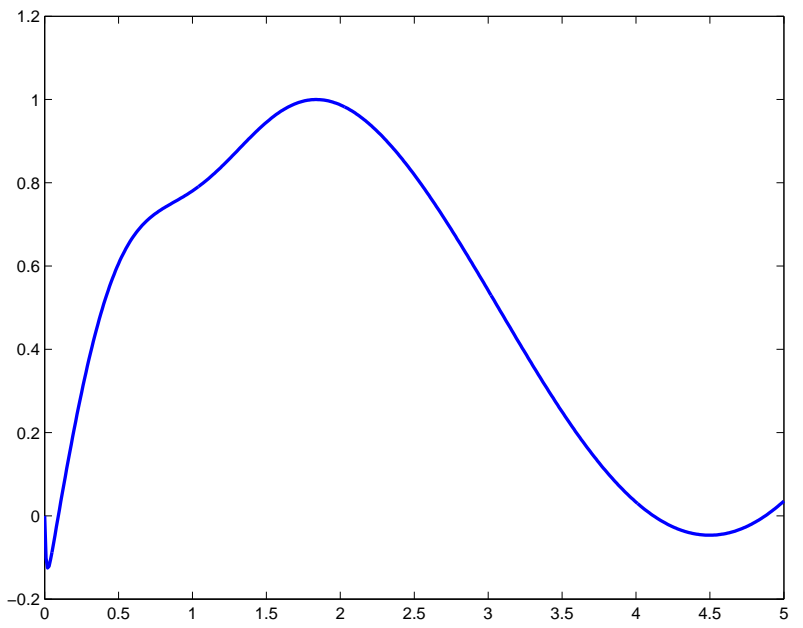

Fig. 2. Input $u(t)$ of the system

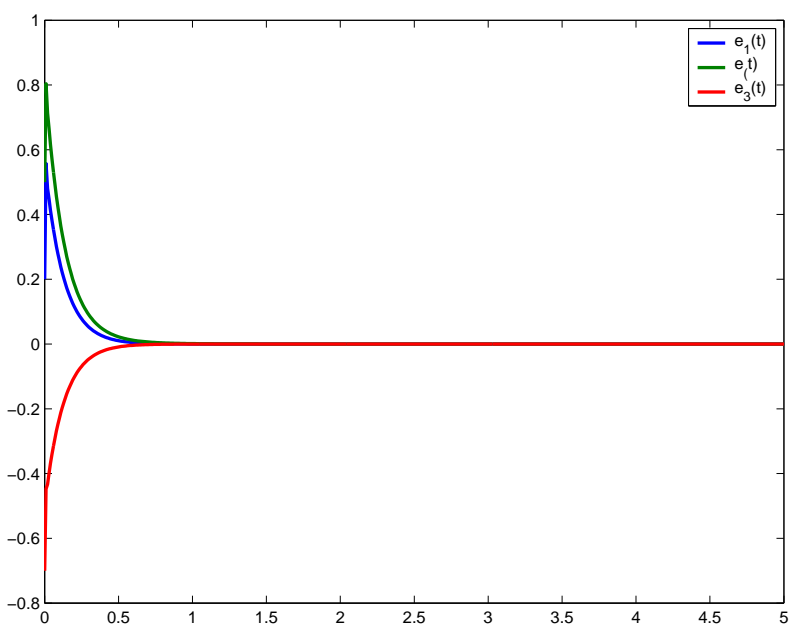

Fig. 3. State estimation error

\section{B. Sensor fault diagnosis}

Let us consider the same system as in the previous subsection. The sensors are now assumed to be affected by faults and noise measurements as described by (46). The faults are given by

$$
\begin{aligned}
& f_{1}(t)= \begin{cases}1 & 2 \leq t \leq 4 \\
0 & \text { elsewhere }\end{cases} \\
& f_{2}(t)= \begin{cases}1 & 6 \leq t \leq 8 \\
0 & \text { elsewhere }\end{cases}
\end{aligned}
$$

The residual signals obtained from the first observer can only detect the sensor fault $f_{2}(t)$ which affects the second 


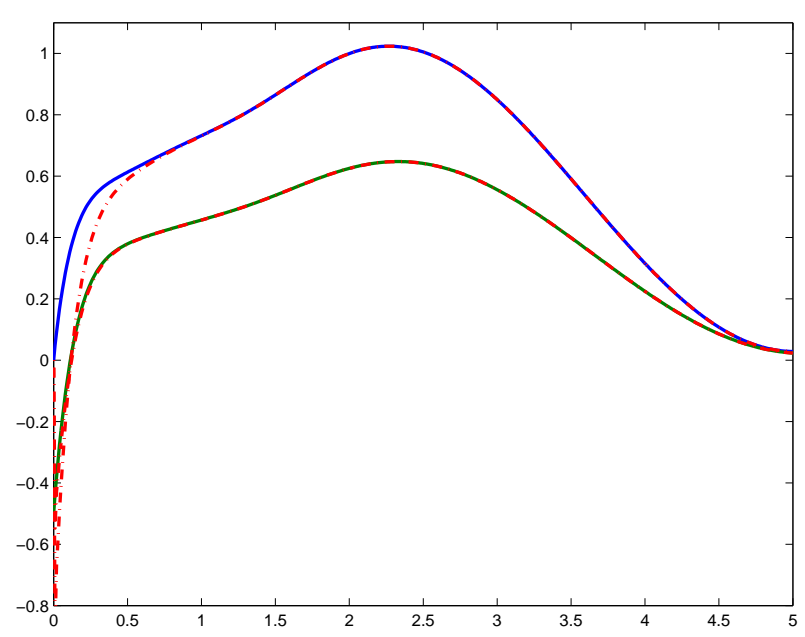

Fig. 4. Actual and estimated outputs

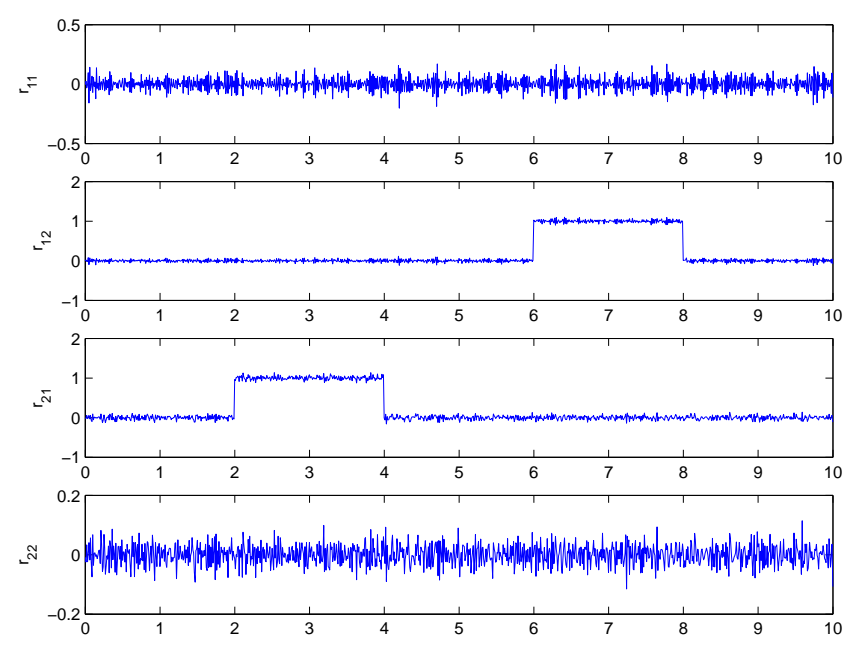

Fig. 5. Residual signals

output $y_{2}(t)$. And the second observer can only detect the fault $f_{1}(t)$ affecting the first output $y_{1}(t)$. The simulation results depicted in the figure 5 show that the first observer is only sensitive to the fault affecting the second sensor and the second one is only sensitive to the fault affecting the first sensor. Then, the diagnosis scheme allows to detect and isolate the sensor faults according to the table 1 .

\section{CONCLUSION}

In this paper, a new approach is proposed to design an observer in order to estimate the state of a nonlinear system described by TS fuzzy model with unmeasurable premise variable. The main idea is to introduce the estimated state in the model and using the convex sum property of the weighting functions, the system is written in the form of uncertain TS model with estimated premise variable. The convergence of the state estimation error is studied using the Lyapunov theory and the $\mathcal{L}_{2}$ approach and the conditions of convergence are given in Linear Matrix Inequality (LMIs). The method is applied successfully to an academic example in order to estimate the states of the system and to construct observer bank to detect and isolate sensor faults. The interest of our approach is the fact that the Lipschitz conditions is not required to construct the observer; thus the weighting functions can be more general, which allows to say that this approach is less restrictive than the approach based on the Lipschitz condition proposed in [5]. The future works will concern the study of the conservativeness of the proposed conditions related to the use of a quadratic Lyapunov function by using other kinds of Lyapunov functions in the one hand, and in the diagnosis problem, the amelioration of fault detection in the presence of disturbances by minimizing the effects of these last and maximizing the effects of the faults on the residual signals, in the other hand.

\section{REFERENCES}

[1] A. Akhenak, M. Chadli, J. Ragot, and D. Maquin. Design of sliding mode unknown input observer for uncertain Takagi-Sugeno model. In 15th Mediterranean Conference on Control and Automation, MED'07, Athens, Greece, 2007.

[2] A. Akhenak, M. Chadli, J. Ragot, and D. Maquin. Fault detection and isolation using sliding mode observer for uncertain Takagi-Sugeno fuzzy model. In 16th Mediterranean Conference on Control and Automation Congress Centre, Ajaccio, France, June 25-27 2008.

[3] P. Bergsten and R. Palm. Thau-Luenberger observers for TS fuzzy systems. In 9th IEEE International Conference on Fuzzy Systems, FUZZ IEEE 2000, San Antonio, TX, USA, 2000.

[4] P. Bergsten, R. Palm, and D. Driankov. Fuzzy observers. In IEEE International Fuzzy Systems Conference, Melbourne Australia, 2001.

[5] P. Bergsten, R. Palm, and D. Driankov. Observers for Takagi-Sugeno fuzzy systems. IEEE Transactions on Systems, Man, and Cybernetics - Part B: Cybernetics, 32(1):114-121, 2002.

[6] S. Boyd, L. El Ghaoui, E. Feron, and V. Balakrishnan. Linear Matrix Inequalities in System and Control Theory. SIAM ed., 1994.

[7] M. Chadli, D. Maquin, and J. Ragot. Non quadratic stability analysis of Takagi-Sugeno systems. In IEEE Conference on Decision and Control, CDC'2002, Las Vegas, Nevada, USA, 2002.

[8] E. Cherrier, J. Ragot, M. Boutayeb, and M. Aziz-Alaoui. Observerbased exponential synchronization of chaotic multimodels. In European Control Conference, ECC'07, 2007.

[9] S. X. Ding. Model-Based Fault Diagnosis Techniques Design Schemes, Algorithms, And Tools. Springer-Verlag, 2008.

[10] M. El-Ghatwary, S.X. Ding, and Z. Gao. Robust fuzzy fault detection for continuous-time nonlinear dynamic systems. In IFAC Symp. SAFEPROCESS, Beijing, China, 2006.

[11] T.M. Guerra, A. Kruszewski, L. Vermeiren, and H. Tirmant. Conditions of output stabilization for nonlinear models in the TakagiSugeno's form. Fuzzy Sets and Systems, 157(9):1248-1259, May 2006.

[12] A. Kruszewski, R. Wang, and T.M. Guerra. Nonquadratic stabilization conditions for a class of uncertain nonlinear discrete time TS fuzzy models: A new approach. Transactions on Automatic Control, IEEE, 53(2):606 - 611, March 2008.

[13] B. Marx, D. Koenig, and J. Ragot. Design of observers for Takagi Sugeno descriptor systems with unknown inputs and application to fault diagnosis. IET Control Theory and Applications, 1:1487-1495, 2007.

[14] R. Orjuela, B. Marx, J. Ragot, and D. Maquin. State estimation for non-linear systems using a decoupled multiple model. Int. J. Modelling, Identification and Control, 4(1):59-67, 2008.

[15] K. Tanaka, T. Hori, and H.O. Wang. A multiple lyapunov function approach to stabilization of fuzzy control systems. IEEE Transactions on Fuzzy Systems, 11(4):582- 589, August 2003.

[16] K. Tanaka, T. Ikeda, and H.O. Wang. Fuzzy regulators and fuzzy observers: Relaxed stability conditions and LMI-based designs. IEEE Transactions on Fuzzy Systems, 6(2):250-265, 1998.

[17] K. Tanaka and H.O. Wang. Fuzzy Control Systems Design and Analysis: A Linear Matrix Inequality Approach. 2001.

[18] J. Yoneyama. $H_{\infty}$ filtering for fuzzy systems with immeasurable premise variables: an uncertain system approach. Fuzzy Sets and Systems, In Press, Accepted Manuscript:-, 2008. 